\title{
Mayores niveles de ECA y Angiotensina II determinados genéticamente, se asocian a menor actividad del eje ECA2/angiotensina-(1-9) y mayor remodelamiento de la pared aórtica de ratas hipertensas.
}

\author{
Jackeline Moya ${ }^{1}$, Ulises Novoa ${ }^{1}$, Nicolás Escudero ${ }^{1}$, Iván Godoy $^{1}$, Mario Chiong ${ }^{2}$, Sergio Lavandero ${ }^{2}$, Jorge Jalii ${ }^{1}$, \\ María Paz Ocaranza ${ }^{1}$. \\ 1 Facultad de Medicina. Pontificia Universidad Católica de Chile. \\ 2 Centro FONDAP CEMC. Universidad de Chile
}

Introducción: El polimorfismo del gen de la enzima convertidora de angiotensina I (ECA) determina mayor actividad de la ECA y mayores niveles de angiotensina (Ang) II. Un polimorfismo similar ha sido descrito en humanos. La ECA2, a través de Ang-(1-9) más que Ang-(1-7), contrarresta los efectos deletéreos de Ang II. Se desconoce si el polimorfismo de la ECA frente a un estímulo hipertensivo modifica el eje ECA2/Ang-(1-9) y determina mayor remodelamiento de la pared aórtica de ratas hipertensas.

Objetivo: Determinar el efecto del polimorfismo del gen de la ECA en la actividad del eje ECA2/Ang-(1-9) y su efecto en el remodelamiento de la pared aórtica secundaria a la hipertensión arterial (HTA) experimental. Métodos: Se usaron ratas macho homocigotas de 150 gr BN y LL. Se indujo HTA por el procedimiento
Goldblatt (GB, 2 K-1clip). Ratas pseudo-operadas se usaron como controles (Sham). A las 6 semanas post cirugía se determinaron en la aorta las actividades de ECA y ECA2, los niveles de Ang II/Ang-(1-9), colágeno tipo I, células positivas para el marcador de inflamación ED1, área y grosor de la túnica media (ATM, GTM).

Resultados: El polimorfismo de la ECA con mayores niveles de ECA y Ang II determinó una mayor disminución de la actividad de ECA2, menores niveles de Ang-(1-9) y mayor remodelamiento de la pared aórtica tanto en animales normotensos como hipertensos.

Conclusión: El polimorfismo de la ECA con mayor actividad de ECA y AngII determina una interregulación de los ejes ECA/AngII y ECA2/Ang-(1-9) lo que se asocia a mayor remodelamiento de la pared aórtica. Fondecyt 1100874.

Correspondencia:

María Paz Ocaranza,

División de Enfermedades Cardiovasculares. Facultad de Medicina. Pontificia Universidad Católica de Chile.

Tel: +562-3543407, Fax: +562-6321924.

E-mail: mocaran@med.puc.cl 


\section{Increased levels of ACE and angiotensin II genetically determi-} ned, are associated with lower ACE2/Angiotensin-(1-9) activity axis and aortic wall increased remodeling in hypertensive rats.

background: The angiotensin converting enzyme (ACE) gene polymorphism determines increased ACE activity and angiotensin (Ang) II levels in Brown Norway rats (BN), compared to Lewis rats (LL). Similar polymorphism has been described in humans. ACE2 through Ang-(1-9) rather than Ang-(1-7) counteracts the deleterious effects of Ang II. It is unknown whether the ACE polymorphism counteracts the ECA2/Ang19 axis and determines increased remodeling of the aortic wall in hypertensive rats.

Objective: To determine the effects of ACE gene polymorphism in the ECA2/Ang1-9 axis activity and its impact on the aortic wall remodeling secondary to hypertension (HT).

Methods: Male homozygous rats $\mathrm{BN}$ and $\mathrm{LL}$ were used. Hypertension was induced by the Goldblatt

\section{Introducción}

En la hipertensión arterial (HTA), la pared aórtica sufre cambios morfométricos y estructurales, proceso denominado remodelamiento vascular (RMV). ${ }^{1}$ Uno de los principales factores fisiopatológicos asociados al desarrollo de la HTA es la sobreactivación de la vía clásica del Sistema Renina Angiotensina (SRA) compuesta por la enzima convertidora de angiotensina I (ECA) y su producto angiotensina (Ang) II. ${ }^{1}$ La Ang II se une a su receptor AT1 (RAT1) activando vías de señalización que desencadena un aumento de la presión arterial (PA) y cambios celulares, no celulares y funcionales en distintos tejidos que incluyen a nivel de la pared aórtica, hipertrofia, fibrosis, inflamación, entre otros, y que conllevan a disfunción endotelial. ${ }^{1,2,3}$

El descubrimiento de la ECA homóloga (ECA2) ${ }^{4,5}$ estableció una vía paralela del SRA. En esta vía, la ECA2 compite con la ECA por la hidrólisis de Ang I para formar Ang-(1-9), 5, 6 además ECA2 transforma Ang II a Ang-(1-7). ${ }^{7}$ Es conocido que Ang-(1-7) presenta acciones opuestas a las de Ang II, al unirse a su receptor MAS, ${ }^{8}$ mediando la vasodilatación y efectos anti-hipertróficos, anti-inflamatorios y anti-fibróticos. ${ }^{8}{ }^{8}$ En relación a la actividad biológica de Ang-(1-9) hay pocos antecedentes disponibles. Nuestro grupo aportó los primeros antecedentes del efecto biológico de Ang-(1-9) en la prevención de la procedure (GB, 2 K-1clip). Pseudo-operated rats were used as controls (Sham). At 6 weeks after surgery, we determined the body weight (BW) and systolic blood pressure (SBP). In aorta, we determined the ACE and ACE2 activities, Ang II/Ang1-9 levels, protein expression of collagen type I, positive cells for ED-1 inflammatory cells and medial thickness (MT) and area (MA) of aortic wall.

Results: ACE polymorphism with higher levels of ACE and Ang II determined a significant decrease of ACE2 activity, Ang-(1-9) levels and aortic wall remodeling in normotensives and hypertensives rats.

Conclusion: ACE polymorphism with increased ACE activity and AngII levels determines a significant inter-regulation between ACE/AngII and ACE2/Ang(1-9) axis which is associated with increased remodeling of the aortic wall. Fondecyt 1100874.

hipertrofia cardiomiocitaria in vivo e in vitro. ${ }^{10}$ Estos hallazgos fueron corroborados por Flores y colaboradores, al establecer que el efecto antihipertrófico de Ang-(1-9) estaría mediado por el receptor tipo 2 de Ang II. ${ }^{11}$

Recientemente, hemos encontrado, en un modelo experimental de HTA por sobrecarga de volumen y en ratas normotensas sham, que la inhibición de RhoA/Rho-kinasa (una vía de señalización que participa en el remodelamiento cardiovascular y renal y también en la regulación de la presión arterial), por fasudil disminuyó la HTA y aumentó la actividad de ECA2 en plasma y pared aórtica. Igualmente, Fasudil disminuyó los niveles plasmáticos de Ang II, aumentó los niveles de Ang-(1-9) sin modificar los niveles de Ang-(1-7)..$^{12}$ Paralelamente, observamos en ratas hipertensas Goldblatt (GB, 2 riñones-1 pinzado, modelo experimental dependiente de Ang II), que el aumento de la presión arterial (PA) y remodelamiento de la pared aórtica se asoció a un aumento significativo de los niveles de Ang II, de la expresión de ARNm y proteína de ECA, junto con una menor expresión de ECA2, y niveles aórticos de Ang (1-9), sin diferencias en los niveles de Angs I y (1-7). ${ }^{13}$ Ambos antecedentes apuntan a que mecanismos de interregulación entre los ejes ECA/Ang II y ECA2/Ang-(1-9) estarían involucrados en la HTA y el remodelamiento de la pared aórtica. Por lo tanto, el objetivo de este estudio fue determinar en un modelo genético de ratas con distintos niveles circulantes de ECA y Ang II,${ }^{14}$ 
la interregulación de los ejes ECA/AngII y ECA/Ang1-9 en la hipertensión y el remodelamiento de la pared aórtica de ratas hipertensas.

\section{Métodos}

El estudio se realizó según la "Guía para el cuidado y uso de animales de laboratorio" publicado por el "National Health Institute" (NIH N $85-23,1985)$ y aprobada por la Comisión de Investigación de la Facultad de Medicina de la Pontificia Universidad Católica de Chile.

1. Modelo experimental: Se usaron ratas homocigotas machos normotensas (peso $150 \pm 10 \mathrm{~g}$ ), de $2^{\circ}$ generación las cuales presentan un polimorfismo en el gen ECA, que determina menor y mayor actividad de ECA y niveles de Ang II para las ratas Lewis (LL) y Brown Norway (BN), respectivamente. ${ }^{15}$ Las ratas fueron randomizadas a operación Goldblatt (GB; 2Riñones-1Clip) para inducir hipertensión arterial. ${ }^{22}$ Como grupo control normotenso (Sham), se usaron ratas sometidas a similares condiciones de estrés. A estos animales se les midió la presión arterial sistólica (PAS) semanalmente por 6 semanas post-cirugía. $\mathrm{Al}$ cabo de este período fueron eutanasiados.

2. Determinación del polimorfismo de la ECA: El ADN se extrajo de leucocitos circulantes de rata, usando resina quelante Chelex 100. Brevemente, 20 $\mu \mathrm{l}$ de sangre (con EDTA) se resuspendieron en $1 \mathrm{ml}$ de agua desionizada estéril y se incubó por 30 min a temperatura $\left(\mathrm{T}^{\circ}\right)$ ambiente con agitación ocasional. La mezcla se centrifugó a $15.000 \mathrm{~g}$ por $3 \mathrm{~min}$ a $\mathrm{T}^{\mathrm{o}}$ ambiente, el sobrenadante se descartó y la pella se resuspendió en $200 \mu \mathrm{l}$ de Chelex 100 al $5 \%$ y se incubó a $56^{\circ} \mathrm{C}$ por $30 \mathrm{~min}$. Luego, se agitó e incubó a $100^{\circ} \mathrm{C}$ por $8 \mathrm{~min}$; posteriormente se agitó en vórtex por $15 \mathrm{seg}$. Finalmente, el ADN se obtuvo en el sobrenadante, después de centrifugar la mezcla a $15.000 \mathrm{~g}$ por 3 min a $25^{\circ} \mathrm{C} .{ }^{16}$ Para la detección del polimorfismo de la ECA se radiomarcó el partidor anti-sentido (RECA-2). La mezcla de reacción contenía: 10 pmoles RECA-2, tampón reacción T4 fosfoquinasa 1X (70mM Tris, $\mathrm{pH}$ 7,6; $10 \mathrm{mM} \mathrm{MgCl} 2,100 \mathrm{mM} \mathrm{KCl}, 1 \mathrm{mM}$ 2-mercaptoetanol), $5 \mathrm{mM}$ Ditiotreiol (DTT), 50 $\mu \mathrm{Ci}$ [ $\gamma$-32P] ATP y $0,3 \mathrm{U} / \mathrm{ml}$ $\mathrm{T} 4$ fosfoquinasa. Se incubó a $37^{\circ} \mathrm{C}$ por 1 hora y se detuvo la reacción a $65^{\circ} \mathrm{C}$ por $10 \mathrm{~min} \cdot{ }^{17} \mathrm{La}$ mezcla de reacción de amplificación en cadena contenía $20 \mu \mathrm{l}$ de ADN, tampón PCR 1x (20 mM Tris, $50 \mathrm{mM} \mathrm{KCl} \mathrm{pH} \mathrm{8,3),} 1 \mathrm{mM} \mathrm{MgCl}$, $100 \mu \mathrm{M}$ dNTP, 10pmoles partidores sentido, 10pmoles partidores anti-sentido radiactivo y $1 \mathrm{UI}$ Taq polimerasa. Las reacciones de denaturación, alineamiento de los partidores y de polimerización se realizaron a través de un termociclador utilizando el siguiente programa de $\mathrm{T}^{\mathrm{o}}: 1$ ciclo por $3 \mathrm{~min}$ a $94^{\circ} \mathrm{C}, 30$ ciclos por $30 \mathrm{seg} 94^{\circ} \mathrm{C}, 30 \mathrm{seg}$ a $65^{\circ} \mathrm{C}$ y 30 seg a $72^{\circ} \mathrm{C}$ y 1 ciclo de 5 min a $72^{\circ} \mathrm{C} .{ }^{18}$
Las secuencias nucleotídicas de los partidores usados son las siguientes 18:

Oligo sentido: 5 ATT ACC ATA GAG GGC AGC AAG ATC 3'

Oligo anti-sentido: 5 CAG ACT TTT CAC CAA TTT TGA CAGC 3

La separación de los fragmentos de ADN amplificados se realizó en geles de acrilamida-bisacrilamida al 6\%, utilizando un aparato de secuenciación por $4 \mathrm{hrs}$ a $30 \mathrm{~W}$. Finalmente, el gel se radioautografió por $16 \mathrm{hrs} \mathrm{a}-80^{\circ} \mathrm{C}$ y se reveló.

3. Presión arterial sistólica: La PAS fue medida semanalmente por pletismografía en la cola de las ratas previamente anestesiadas. ${ }^{19}$

4. Obtención del tejido Aórtico: Al final del tratamiento, los animales fueron anestesiados con Ketamina $(35 \mathrm{mg} / \mathrm{kg})$ y Xilacina $(7 \mathrm{mg} / \mathrm{kg})$ por vía intra-peritoneal. Las aortas fueron extraídas y lavadas en suero fisiológico, una parte del tejido fue fijado en bouin e incluido en parafina. El resto del tejido fue conservado a $-80^{\circ} \mathrm{C}$.

\section{Actividad de ECA y ECA-2 en la pared aórtica}

La actividad de la ECA se determinó tal como lo describió Oliveri et al ${ }^{20}$ y se expresó en U/mg proteina (1U: nmoles HL/min). Para la medición de la actividad de ECA-2 se usó como sustrato el péptido 7-metil coumarina (Mea) -R-P-P-G-F-S-A-F-K(Dnp)-OH_(R\&Dsystems), el cual posee un grupo apagador (Dnp) de la fluorescencia descrito por Jonhson y Ahn ${ }^{21}$. Brevemente, el ensayo de actividad enzimática se realizó en un volumen total de $100 \mu \mathrm{L}$ de tampón de reacción en el cual se adicionaron $20 \mu \mathrm{L}$ de de plasma o $10 \mu \mathrm{L}$ de homogenizado de VI, 10 uM de sustrato,ßmM de enalapril y tampón de reacción (50 mM Tris-HCI, pH 7,4, 0,2 mM NaCI). Como control se usó igual mezcla de reacción a la cual se le adicionó 10 $\mathrm{mM}$ de EDTA. La mezcla se incubó a $37^{\circ} \mathrm{C}$ por $30 \mathrm{~min}$ y la reacción enzimática fue detenida calentando a $100^{\circ} \mathrm{C}$ por $5 \mathrm{~min}^{19}$. Posteriormente las muestras se centrifugaron a $10.000 \mathrm{~g}$ por $10 \mathrm{~min}$. La actividad en aorta se expresó en U (pmoles de 7 metil coumarina/min)/mg proteína).

\section{Niveles de angiotensinas en la pared aórtica.}

La aorta se separó y se homogenizó en $5 \mathrm{~mL}$ de $4 \mathrm{M}$ de tiocianato de guanidinio (GTC). Los homogenizados fueron almacenados a $-80^{\circ} \mathrm{C}$ hasta ser extraídos en minicolumnas Sep-Pak C18. Las Angs fueron separadas por HPLC y cuantificadas por radioinmunoensayo (RIA) usando anticuerpos policlonales dirigido contra los extremos aminoterminales donado por el Dr DJ Campbell (St, Vincenfs Institute of Medical Research, University of Melbourne, Fitzroy) $)^{19,22 .}$

\section{Hipertrofia de la pared aórtica:}

Se utilizaron cortes transversales de aorta (Ao) de $5 \mu \mathrm{m}$ 
de espesor teñidos con hematoxilina-eosina. Las imágenes fueron tomadas mediante una cámara acoplada a un microscopio (Nikon) y cuantificadas utilizando un software (Nis-Element). Se registró el área del lumen (AL, $\mathrm{mm} 2$ ) y total (AT, mm2) del anillo aórtico (4X). El área de la túnica media (ATM, mm2), se obtuvo de la diferencia entre AT y AL. ${ }^{23}$ Para determinar el grosor de la túnica media (GTM, $\mu \mathrm{m}$ ) se realizaron 10 mediciones por cada sección de anillo aórtico (40X). ${ }^{13}$ Se analizaron al menos 3 anillos aórticos por animal, los que se seleccionaron aleatoriamente.

\section{Contenido de colágeno I de la pared aórtica:}

Se utilizaron $50 \mathrm{mg}$ de tejido aórtico que fueron homogenizados en tampón de lisis y centrifugado a $10000 \mathrm{~g}$ por 30 min a $4^{\circ} \mathrm{C} \cdot{ }^{24} \mathrm{El}$ contenido de proteínas se midió por el método de Bradford. ${ }^{25}$ Para el Western blot se utilizaron $50 \mu \mathrm{g}$ de proteína total de Ao, siguiendo el protocolo descrito por Rivera et al., 2007. ${ }^{24}$ Utilizando como anticuerpo primario, anti Col I (dilución 1:3000, AB749 Chemicon) y anticuerpo segundario, anti-IgG de conejo conjugado a peroxidasa (dilución 1:10000, Pierce 31460). Como control de carga se utilizó $\beta$-actina, empleando un anticuerpo anti $\beta$-actina (Sigma A5316) con una dilución de 1:5000 y como anticuerpo secundario, anti-IgG de ratón conjugado a peroxidasa (Piarce 31457) en dilución 1: 10.000.

\section{Inflamación de la pared aórtica:}

Cortes transversales de $4 \mu \mathrm{m}$ de grosor de Ao fueron desparafinados para luego realizar desenmascaramiento antigénico del tejido con EDTA $1 \mathrm{mM}$ a pH $8 .{ }^{26} \mathrm{La}$ inmunomarcación se realizó con el Kit DAKO K0679. Los cortes se incubaron con anti-ED1 de macrófagos de rata (Serotec MCA341R) en una dilución 1/200 toda noche en una cámara húmeda a $4^{\circ} \mathrm{C}$. Posteriormente los tejidos se lavaron y se incubaron con el anticuerpo secundario biotinilado durante $30 \mathrm{~min} \mathrm{~T}^{\mathrm{o}}$ ambiente. Como método de detección se utilizó la técnica de Diaminobenzidina (Kit DAKO) y contratinción con hematoxilina. El número de células marcadas con ED-1 se midieron de forma cuantitativa, ${ }^{27}$ evaluando la razón entre el número total de células positivas para ED1 y el área de anillo aórtico (ED1/A, mm²).

\section{Análisis de resultados:}

Los datos obtenidos se expresaron como promedio \pm error estándar de la media (SEM). Cada grupo experimental estuvo constituido por 4-8 animales. Las comparaciones entre los grupos se realizaron mediante Kruskal-Wallis, seguido de un análisis de Mann-Whitney. Se consideraron diferencias significativas con $\mathrm{p} \leq 0,05$.

\section{Resultados}

1. Efecto del polimorfismo de la ECA en la PAS y Masa Cardíaca: En los modelos LL y BN Sham, la PAS se

mantuvo en condiciones de normotensión, obteniendo un promedio de $109 \pm 1$ y $111 \pm 1 \mathrm{mmHg}$, respectivamente, durante las 6 semanas de ensayo sin diferencias por el efecto del polimorfismo de la ECA (Fig. 1). Post-cirugía las ratas GB aumentaron la PAS a partir de la primera semana, observándose un aumento significativo de la PAS en un $23 \%$ para las ratas LL-GB $(134 \pm 8 \mathrm{v} / \mathrm{s} 109 \pm 1$ $\mathrm{mmHg}, \mathrm{p}=0,019$, v/s LL-Sham, Fig. 1) y BN-GB (138 \pm $6 \mathrm{v} / \mathrm{s} 112 \pm 4 \mathrm{mmHg}, \mathrm{p}=0,003$, v/s BN-Sham, Fig. 1). La PAS siguió aumentando significativamente durante las 6 semanas, llegando a registrar a la sexta semana un aumento de un $50 \%$ en el modelo LL-GB $(161 \pm 3 \mathrm{v} / \mathrm{s} 107 \pm 4$ $\mathrm{mmHg}, \mathrm{p} \leq 0,0001$, Fig. 1) y un $45 \%$ en el grupo BN-GB $(160 \pm 2 \mathrm{v} / \mathrm{s} 110 \pm 2 \mathrm{mmHg}, \mathrm{p} \leq 0,0001$, Fig. 1) respecto a sus respectivos controles sham. No se encontraron diferencias significativas en la PAS entre las ratas GB LL y $\mathrm{BN}$ (Fig. 1). En condición de normotensión la MV de las ratas $\mathrm{BN}$ fue un $13 \%$ mayor que las ratas LL (Tabla1). En respuesta al estímulo hipertensivo, la MV aumentó significativamente un $48 \%$ en las ratas LL-GB y un $30 \%$ en las ratas BN-GB (Tabla 1) versus sus controles normotensos, sin diferencias entre ambos polimorfismos.

Figura 1. Presión arterial sistólica en ratas homocigotos LL y BN normotensas e hipertensas.

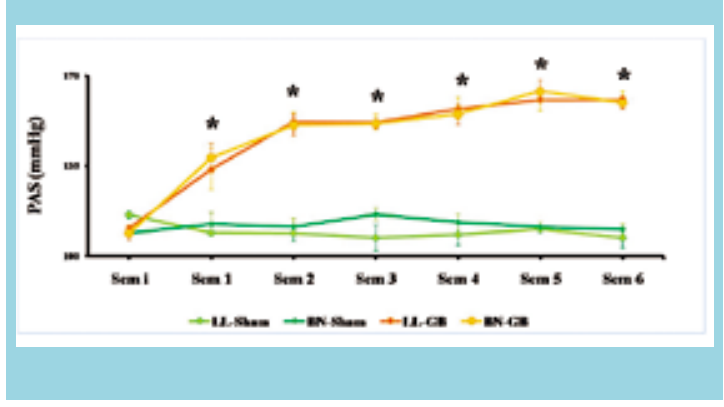

Abreviaturas: $P A S=$ presión arterial sistólica, Sham = ratas normotensas, $G B=$ ratas hipertensas, $L L=$ ratas Lewis con menor actividad de la ECA y niveles de Ang II, BN = ratas Brown Norway con mayor actividad de la ECA y niveles de Ang II, Sem = Semana $y i=$ Inicial. Los resultados se graficaron según el promedio \pm $S E M, N=7-8$ por grupo, $* p \leq 0,05$ Sham, después de KruskalWallis, seguido de un análisis de Mann-Whitney para comparación entre grupos.

2. Actividad enzimática de ECA y ECA2 en la pared aórtica: En condición de normotensión el polimorfismo genético de la ECA determinó en la pared aórtica de ratas BNSham una actividad de la ECA $20 \%$ mayor, respecto a las ratas LL-Sham (78 \pm 5 vs $65 \pm 3$, Fig. 2A). En condición de hipertensión la actividad de la ECA aumentó significativamente en un $100 \%$, tanto en las ratas BN y LL, respecto a sus controles Sham BN y LL, respectivamente (Fig. 2A). 
Sin embargo, el polimorfismo de la ECA determinó que las ratas BN-GB presentarán un aumento significativo de la actividad de la $\operatorname{ECA}(20 \%, \mathrm{p}<0,05)$, respecto a las ratas GB-LL (156 \pm 7 vs $130 \pm 6$, Fig. 2A).

La actividad enzimática de la ECA-2 fue un $46 \%$ menor en las ratas BN-Sham versus LL-Sham $(0.28 \pm 0.04$ vs $0.15 \pm 0.03$; Fig. 2B). La HTA disminuyó significativamente la actividad de la ECA-2 (40\%) en ambos modelos GB con respecto a sus controles Sham (Fig. 2B). El polimorfismo de la ECA determinó que las ratas hipertensas $\mathrm{BN}$ presentaran significativamente menor actividad de la ECA-2 (-44\%, 0.09 \pm 0.01 vs $0.16 \pm 0.02$; Fig. $2 B)$, respecto a las ratas hipertensas LL.

3. Efecto del polimorfismo de la ECA en los niveles de angiotensinas de la pared aórtica: Los niveles de Ang II en la pared aórtica de las ratas normotensas BN fueron un $23 \%$ mayor que las ratas LL-Sham $(79 \pm 3$ vs $60 \pm 3$, Tabla 1). La hipertensión aumentó significativamente los niveles de Ang II tanto en las ratas LL y BN respecto a sus controles normotensos ( $\mathrm{p}<0.05$,Tabla 1). Igualmente, a lo observado en la actividad de la ECA de la pared aórtica, las ratas hipertensas BN mostraron mayores niveles de Ang II $(14 \%, \mathrm{p}<0.05)$, respecto a las ratas LL-GB $(103 \pm 3$ vs $89 \pm 3$, Tabla 1$)$.

\begin{tabular}{|c|c|c|c|c|}
\hline & LL-Sham & LL-GB & BN-Sham & BN-GB \\
\hline$n$ & 8 & 8 & 8 & 8 \\
\hline PC (gr) & $225 \pm 12$ & $248 \pm 14$ & $238 \pm 3$ & $241 \pm 8$ \\
\hline $\mathrm{MC}(\mathrm{gr})$ & $0,68 \pm 0,01$ & $1,01 \pm 0,03^{*}$ & $0,77 \pm 0,01^{\&}$ & $1,0 \pm 0,02$ \\
\hline Ang II (fmoles/ml) & $60 \pm 3$ & $89 \pm 3^{*}$ & $79 \pm 3^{\&}$ & $103 \pm 3^{\star \$} \$$ \\
\hline Ang-(1-9)(fmoles/ml) & $18,5 \pm 1,3$ & $13,9 \pm 0,7^{\star}$ & $14,3 \pm 0,7^{\&}$ & $164 \pm 4^{* \$}$ \\
\hline
\end{tabular}

Se midió peso corporal (PC), masa cardíaca (MC), Angiotensina II (Ang II) y Angiotensina-(1-9) [Ang-(1-9)] para los distintos grupos experimentales. Abreviaturas: Sham = ratas normotensas, $G B=$ ratas hipertensas, $L L=$ ratas Lewis con menor actividad de la ECA y $B N=$ ratas Brown Norway con mayor actividad de la ECA. Los datos fueron expresados según Promedio \pm SEM. $N=4-8$, *p $\leq$ 0,05 Sham, \# $p \leq 0,05 G B$, \& $p \leq 0,05$ LL-Sham, $\$ p \leq 0,05 L L-G B$, después de Kruskal-Wallis, seguido de un análisis de Mann-Whitney para comparación entre grupos.

En condición de normotensión, los niveles aórticos de Ang-(1-9) en las ratas normotensas BN fueron un $23 \%$ menor respecto a las ratas LL $(14,3 \pm 0.7$ vs $18,5 \pm 1,3$; Tabla1). Las ratas hipertensas mostraron una disminución significativa de los niveles de Ang-(1-9) con respecto a sus controles normotensos (Tabla 1). Las ratas hipertensas BN mostraron un 35\% menores niveles de Ang-(1-9) que las ratas LL-GB $(10,2 \pm 0,5$ vs $13,9 \pm 0.7$, Tabla 1$)$.
Figura 2. Actividad de ECA y ECA-2 en la pared aórtica:

A)

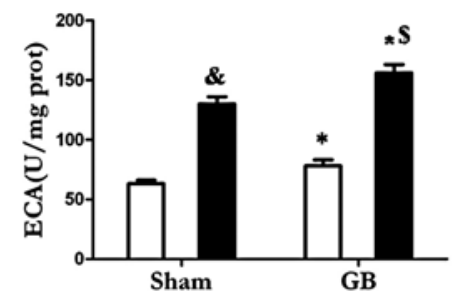

B)

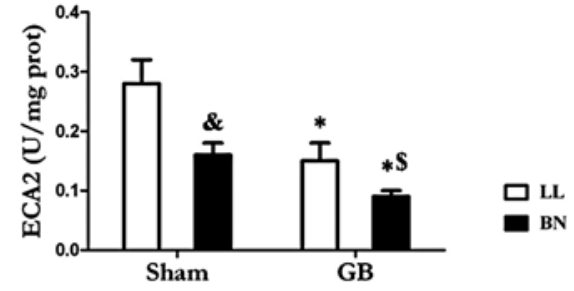

Abreviaciones: Sham $=$ ratas normotensas, $G B=$ ratas hipertensas, $L L=$ ratas Lewis con menor actividad de la $E C A$ y $B N=$ ratas Brown Norway con mayor actividad de la ECA. A) Actividad de ECA (U: nmoles e histidil-leucinalmg proteína) en la pared aórtica. B) Actividad de ECA-2 (U: nmoles de 7-metixocoumarina/mg proteína) en la pared aórtica. Los datos fueron graficados según el promedio \pm SEM, $N=7-8, \& p \leq 0,05$ LL-Sham, * $p \leq 0,05$ Sham y \$ p $\leq$ 0,05 LL-GB, después de Kruskal-Wallis, seguido de un análisis de Mann-Whitney para comparación entre grupos.

4. Polimorfismo del gen de la ECA en la hipertrofia de la pared aórtica: En ratas normotensas BN y LL, el polimorfismo de la ECA no determinó diferencias en el ATM $(0,360 \pm 0,030 \mathrm{v} / \mathrm{s} 0,338 \pm 0,017 \mathrm{~mm} 2$, Fig. 3 A y B $)$ y GTM $(64,78 \pm 3,34 \mu \mathrm{m}$ v/s 65,20 $\pm 1,84 \mu \mathrm{m}$, Fig. 3 A y C). En ratas hipertensas, se encontró un aumento significativo del ATM de un 50\% en las ratas LL ( $p=0,002$, Fig. 3 A y B) y de un $74 \%$ en las ratas $\mathrm{BN}$ ( $p=0,001$, Fig2 A y B), respecto a su controles normotensos. Igualmente, el GTM aumentó un 36\% en las ratas hipertensas LL (p = 0,002 , Fig. 3 A y C) y un $62 \%$ en las ratas hipertensas BN ( $p=0,001$, Fig. 3 A y $C$ ) versus sus controles sham. El polimorfismo de la ECA determinó que las ratas BN-GB presentarán mayor ATM $(0,589 \pm 0,027$ v/s $0,505 \pm 0,013$ $\mathrm{mm} 2, \mathrm{p}=0,022$, Fig. 3 A y B) y GTM $(105,50 \pm 4,09 \mu \mathrm{m}$ $\mathrm{v} / \mathrm{s} 87,85 \pm 0,86 \mu \mathrm{m}, \mathrm{p}=0,001$, Fig. 3 A y C) respecto a las ratas LL-GB.

5. Efecto del polimorfismo de la ECA en la fibrosis vascular: En condición de normotensión los niveles proteicos de Col I fueron significativamente mayores en las ratas $\mathrm{BN}$ versus $\mathrm{LL}(2,08 \pm 0,37 \mathrm{v} / \mathrm{s} 1 \pm 0,25, \mathrm{p}=0,05$, Fig. 4 A y B). Frente a una condición de hipertensión, el nivel proteico de Col I en las ratas LL y BN aumentó significa- 
Figura 3. Hipertrofia de la pared aórtica de ratas homocigotos normotensas e hipertensas.

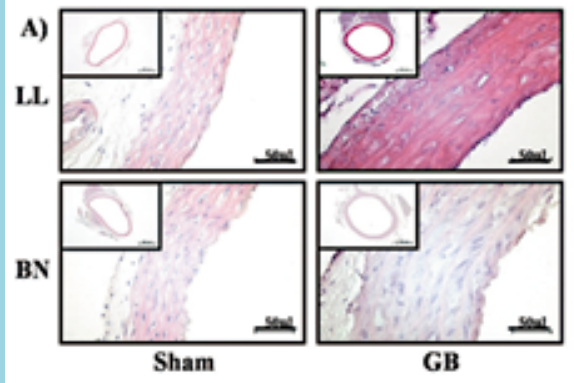

B)

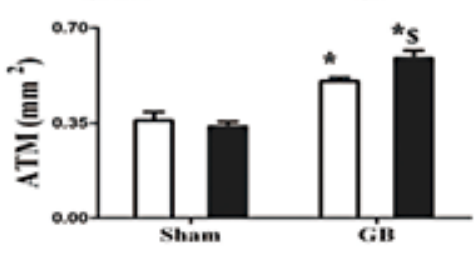

C)

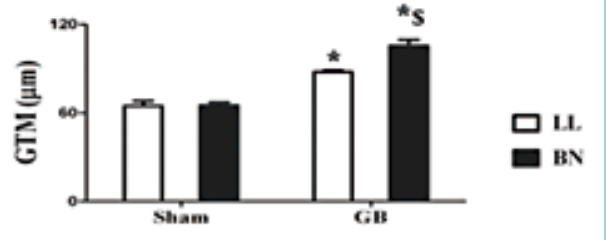

Abreviaturas: $A T M=$ Área de la túnica media, $G T M=$ Grosor de la túnica media, Sham = ratas normotensas, $G B=$ ratas hiper tensas, $L L=$ ratas Lewis con menor actividad de la ECA y $B N=$ ratas Brown Norway con mayor actividad de la ECA. A) Cortes transversales de aorta teñidos con $H$-E. Se graficó el ATM (B) y la razón GTM (C) entre los grupos Sham y GB. Los datos fueron graficados según el promedio \pm SEM. $N=7-8$, * $p \leq 0,05$ Sham y $\$$ $p \leq 0,05$ LL-GB, después de Kruskal-Wallis, seguido de un análisis de Mann-Whitney para comparación entre grupos.

tivamente respecto a sus controles normotensos LL y BN $(4,98 \pm 0,20 \mathrm{v} / \mathrm{s} 1 \pm 0,25, \mathrm{p}=0,001$ y 7,83 $\pm 1 \mathrm{v} / \mathrm{s} 2,08 \pm$ $0,37, \mathrm{p} \leq 0,0001$ respectivamente, Fig. 4 A y B). El polimorfismo de la ECA determinó mayores niveles proteicos de Col I en las ratas BN-GB v/s LL-GB (7,83 \pm 1 v/s 4,98 $\pm 0,20, p=0,011$, Fig. 4 A y B).

6. Polimorfismo de la ECA en la inflamación de la pared aórtica: En ratas normotensas BN, se observó un aumento significativo del $60 \%$ en la razón entre las células tota-
Figura 4. Niveles de colágeno I en la pared aórtica en ratas homocigotos LL y BN normotensas e hipertensas.

A)

Col I
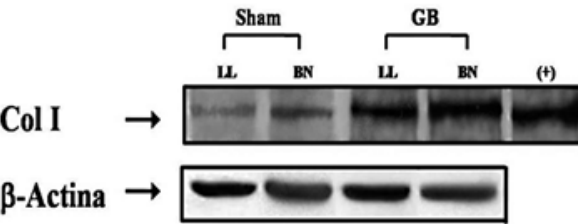

B)

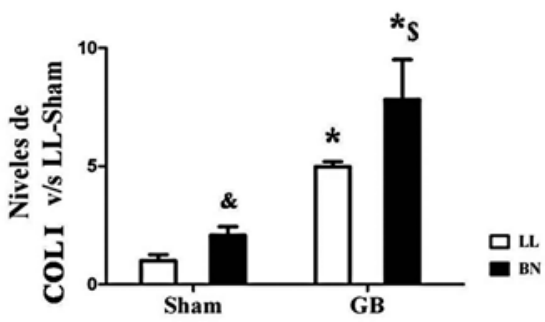

Abreviaturas: Col I = colágeno I, Sham = ratas normotensas, $G B=$ ratas hipertensas, $L L=$ ratas Lewis con menor actividad de la ECA y niveles de Ang II y BN = ratas Brown Norway con mayor actividad de la ECA y niveles de Ang II. A) Diferencias en los niveles proteicos de Col I. B) Gráfico de los niveles de Col I $v / s$ LL-Sham entre los modelos normotensos e hipertensos. Los datos fueron graficados según el promedio $\pm S E M, N=7-8$, \& $p \leq 0,05$ LL-Sham, *p $\leq 0,05$ Sham, y $\$ p \leq 0,05$ LL-GB después de Kruskal-Wallis, seguido de un análisis de Mann-Whitney para comparación entre grupos.

les positivas para ED1 respecto al área del anillo aórtico (ED1/A) respecto a los animales LL ( $p=0,005$, Fig. 5 A y B). La hipertensión aumentó significativamente la razón ED1/A en las ratas LL $\left(918 \pm 117\right.$ v/s $246 \pm 27 \mathrm{~N}^{\circ}$ células/lmm2, p $\leq 0,0001$, Fig. 5 A y B) y BN (1422 \pm 192 v/s $394 \pm 32 \mathrm{~N}^{\circ}$ células/mm2, p = 0,001, Fig. 5 A y B) respecto a sus controles. El polimorfismo de la ECA determinó un aumento de un 55\% en la razón ED1/A de las ratas BN-GB v/s LL-GB ( $\mathrm{p}=0,029$, Fig. 5 A y B). 
Figura 5. Infiltración de macrófagos en la pared aórtica de ratas homicigotos normotensas e hipertensas.
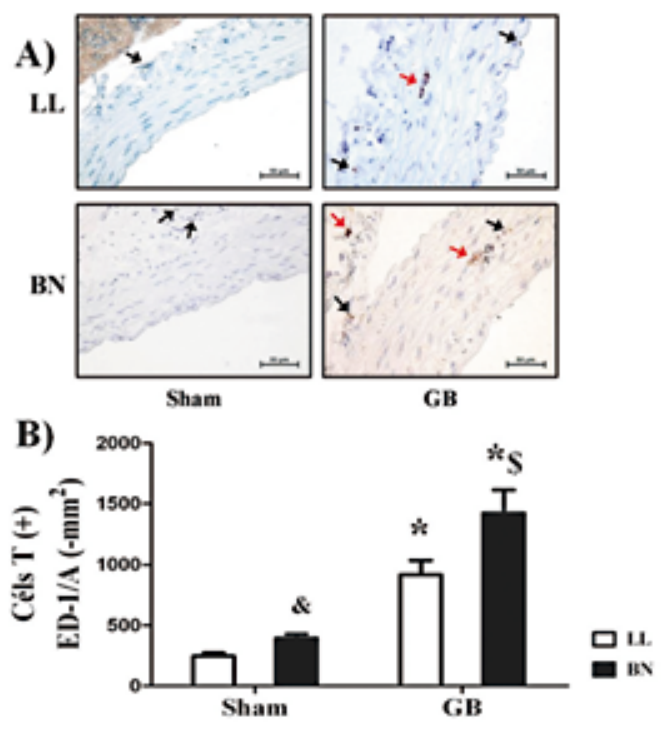

Abreviaturas: Cels $T(+) E D-1 / A=$ Razón entre el número de células totales positivas para ED-1 y el área de anillo aórtico, Sham = ratas normotensas, $G B=$ ratas hipertensas, $L L=$ ratas Lewis con menor actividad de la ECA y niveles de Ang II, BN = ratas Brown Norway que presentan mayor actividad de la ECA y niveles de Ang $I I, \rightarrow=$ céls (+) ED-1 $y \rightarrow=$ cumulo de céls (+) ED-1.A) Inmunohistiquímica de cortes de la pared aórtica rebelados con Diaminobenzidina y contratinción con hematoxilina. Se graficó la razón Cels $T(+)$ ED-1/A (B) entre los modelos Sham y GB. Los datos fueron graficados según el promedio $\pm S E M, N=7-8$, $\& p \leq 0,05 \mathrm{LL}$-Sham, ${ }^{*} p \leq 0,05$ Sham y $\$ p \leq 0,05 \mathrm{LL}$-GB, después de Kruskal-Wallis, seguido de un análisis de Mann-Whitney para comparación entre grupos.

\section{Discusión}

Nuestros resultados muestran que: a) En condiciones de normotensión, una mayor actividad del eje ECA/Ang II -determinada genéticamente- se asocia a una menor actividad del eje ECA2/Ang-(1-9) y mayor contenido de colágeno I e inflamación en la pared aórtica. b) En la hipertensión el eje ECA/Ang II aumenta significativamente, mientras el eje ECA2/Ang-(1-9) se contrarregula negativamente lo que se asocia a mayor remodelamiento de la pared aórtica. Este efecto fue significativamente mayor en los animales con polimorfismo de la ECA que determina mayor actividad de ECA y niveles de Ang II.

Previamente, hemos encontrado en la pared aórtica de ratas normotensas que el polimorfismo de la ECA con mayor actividad de la ECA y mayores niveles circulantes de Ang II (ratas BN) determina mayor estrés oxidativo medido por una mayor actividad de la $\mathrm{NAD}(\mathrm{P}) \mathrm{H}$ oxidasa y producción de aniones superóxido; efecto que fue mediado por el receptor de tipo 1 (RAT1) de Ang II. ${ }^{28}$ Junto con esto, se encontró mayor actividad de la vía transduccional RhoA/Rho-kinasa (una vía de señalización que activada por Ang II, entre otros, que participa en el remodelado cardiovascular y renal, y también en la regulación de la presión arterial), lo que se asoció a mayor expresión de proteínas TGF-beta1, PAI-1 MCP-1, que contribuyen al remodelamiento de la pared aórtica. ${ }^{24}$

Nuestros resultados muestran que en condiciones de normotensión, el polimorfismo de la ECA no solo determinaría mayor actividad del eje ECA/Ang II sino que también menor actividad del eje ECA2/Ang-(1-9),- eje que contrarregula las acciones deletéreas de la Ang II-. Al respecto, encontramos que menor actividad del eje ECA2/Ang(1-9) se asociaría al mayor remodelamiento de la pared aórtica de las ratas $B N$ versus $L$.

Hasta la fecha se han establecido dos ejes primordiales en el balance fisiológico del SRA, el eje vasoconstrictor, ECA-AngII-RAT1 y el eje vasodilatador, ECA2Ang-(1-7)-receptor MAS. ${ }^{29}$ Los primeros antecedentes respecto a la contrarregulación del eje ECA/Ang II y ECA2/Ang-(1-9) fueron aportados por nuestro grupo de investigación en un modelo de ratas infartadas (IAM) de 8 semanas post cirugía. ${ }^{19} \mathrm{Al}$ respecto, encontramos: a) A nivel circulante y en ventrículo izquierdo, mayor actividad de ECA y menor actividad de ECA2.b) La administración diaria del inhibidor de ECA, enalapril, o antagonista del receptor de Ang II, candesartán, por 8 semanas post IAM, previno la hipertrofia ventricular izquierda, disminuyó la actividad de la ECA y aumentó la actividad de la ECA2.c) Los niveles plasmáticos de Ang-(1-9) aumentaron significativamente cuando las ratas IAM o sham fueron tratadas con enalapril o candesartán, sin cambios en los niveles circulantes de Ang-(1-7). Basado en estos antecedentes, se propuso en este modelo experimental, que los niveles de Ang-(1-9) más que Ang-(1-7) contrarregularían las acciones de Ang II. ${ }^{19}$

Recientemente, en ratas IAM aleatorizadas a recibir vehículo, enalapril o candesartán por 8 semanas, Ocaranza et al, observaron que ambas drogas previnieron la hipertrofia ventricular izquierda y aumentaron la actividad de ECA2 y los niveles circulantes de Ang-(1-9), niveles que correlacionaron negativamente con diversos marcadores de hipertrofia ventricular, independiente de la disminución de la presión arterial. Mientras que, los niveles de Ang-(17), Ang II y bradicininas no correlacionaron. ${ }^{10} \mathrm{Al}$ evaluar el efecto de la administración crónica de Ang-(1-9) por mini bombas osmóticas en ratas infartadas por un período de 2 semanas se observó disminución de los niveles circulantes de Ang II, inhibición de la actividad de la ECA 
y prevención del desarrollo de hipertrofia ventricular y cardiomiocitaria in vivo. ${ }^{10}$ Este efecto de Ang-(1-9) fue directo y no mediado por la conversión de Ang-(1-9) a Ang-(1-7). ${ }^{10}$ En experimentos in vitro, con cardiomiocitos incubados con norepinefrina $(10 \mu \mathrm{M})$ o con IGF-1 (10 $\mathrm{nM}$ ), Ang (1-9) también previno la hipertrofia, efecto que no fue modificado por la coincubación de Ang-(1-9) y A779. ${ }^{10}$ Estos resultados fueron confirmados por Flores et al, que estudiaron el efecto de Ang-(1-9) en células de ratas neonatas $\mathrm{H} 9 \mathrm{c} 2 \mathrm{y}$ en cardiomiocitos del ventrículo izquierdo de conejo. ${ }^{11}$ La hipertrofia cardiomicitaria se estimuló con Ang II o vasopresina, aumentando significativamente el tamaño celular como también la expresión de marcadores de hipertrofia cardiomiocitaria. Tanto Ang(1-9) como Ang-(1-7) disminuyeron el tamaño celular y la expresión de marcadores de hipertrofia ventricular. Este efecto de Ang-(1-9) no fue inhibido por captopril, aportando a la evidencia previa que Ang-(1-9) actúa independientemente de Ang-(1-7) y que probablemente su mecanismo de acción sería mediado por el receptor tipo 2 de Ang II (RAT2). Al respecto estudios de unión radioligando demostraron que Ang-(1-9) es capaz de unirse al RAT2 (pKi=6.28 \pm 0.1$).{ }^{11}$ Todos estos hallazgos soportan la hipótesis que, en la vía paralela del SRA, la ECA2 a través de la Ang-(1-9) y no de Ang-(1-7), podría actuar como un contrarregulador de la vía clásica del SRA, donde la ECA cataliza la formación de Ang II.

Varios estudios señalan que el genotipo de la ECA está asociado a alteraciones cardíacas como la HTA, restenosis arterial coronaria, etc, entre otras. ${ }^{30}$ En general, los resultados de estos estudios clínicos, poblacionales y experimentales han sido controversiales respecto a si este polimorfismo contribuye al desarrollo de hipertensión arterial. ${ }^{31}$ Resultados previos del laboratorio ${ }^{14}$ han mostrado que frente a un estímulo hipertensivo, el polimorfismo de la ECA que determina mayores niveles de ECA y Ang II, alcanza mayor presión arterial sistólica. Igualmente, en humanos se encontró una correlación entre hipertensión arterial y polimorfismo deleción-deleción (DD, similar a ratas $\mathrm{BN}$ ) en muestras poblacionales grandes. ${ }^{32}$ Además, se ha publicado que el polimorfismo del gen de la ECA que determina mayores niveles de ECA y Ang II podría inducir respuestas o riesgos diferentes en relación con estímulos fisiológicos y patológicos. Al respecto, Challah et al encontraron que ratas con mayor actividad de la ECA (ratas BN) desarrollaron mayor proliferación de la neoíntima por daño cardíaco en comparación con ratas con menor actividad de al ECA. ${ }^{33}$ Nuestros resultados muestran que frente al estímulo hipertensivo las ratas BN-GB mostraron, un aumento del remodelamiento de la pared aórtica, respecto las ratas hipertensas LL-GB. Estos resultados apuntan a que mayores niveles de ECA y Ang II se asocian a un mayor remodelamiento vascular secundario a la hipertensión. Es conocido que la Ang II induce hipertrofia de la pared aórtica por medio de un incremento de la presión arterial y por lo tanto de estímulos mecánicos que gatillan una mayor expresión de PDGF-A, que podría actuar como mediador de la respuesta hipertrófica vascular. ${ }^{34}$ Además, varios estudios han demostrado que Ang II induce fibrosis e inflamación vascular. ${ }^{35,36}$ Estos efectos son mediante una compleja interacción de vías de señalización intracelular que involucran fosfolipasas, factores de transcripción de genes y activación de tirosinas kinasas. ${ }^{37}$

En vasos, existe abundante información respecto a ECA2, Ang-(1-7) y su receptor Mas. La ECA2 vascular es funcionalmente activa y genera Ang-(1-7) de Ang II. Ang-(1-7) se encuentra en el endotelio y pared vascular ${ }^{38}$, 39,40 y estudios inmunohistoquímicos muestran importante presencia en el tejido de la adventicia perivascular aórtico. ${ }^{41,42}$ Ang-(1-7), se une a su receptor Mas en células endoteliales, se opone a las acciones de Ang II mediando, vasodilatación, inhibición del crecimiento, respuesta anti-inflamatoria y efectos antiarritmogénicos y antitrombóticos, ${ }^{43,44}$ a través de la producción de NO derivada de la NO sintetasa, activación de proteína tirosina fosfatasa, menor activación de MAPK e inhibición de especies reactivas al oxígeno derivadas de NADPH. ${ }^{45,46}$ La sobrexpresión de ECA2 en la pared vascular de ratas espontáneamente hipertensas se asoció a mayor función endotelial y menor desarrollo de hipertensión. ${ }^{45}$ Ang-(1-7)-Mas puede hetero-oligomerizar con RAT1 y por lo tanto, inhibir las acciones de Ang II. La mayoría de la información disponible apuntan a que en la vasculatura, el eje ECA2/Ang-(17)/Mas contrarregularía al eje ECA/Ang II/RAT, ${ }^{46}$ aunque cierta evidencia indica que bajo ciertas condiciones la Ang-(1-7) podría promover fibrosis e inflamación. ${ }^{47,48}$

Nuestros resultados muestran que, frente a un estímulo hipertensivo la actividad del eje clásico del SRA a través de la actividad de la ECA y niveles de Ang II aumentaron significativamente. Junto con esto, la actividad de la vía ECA2/Ang-(1-9) disminuyó significativamente lo que se asoció a mayor remodelamiento de la pared aórtica. El polimorfismo de la ECA determinó en los animales hipertensos con mayor actividad de ECA y niveles de Ang II mayor disminución de la actividad de la ECA2 y niveles de Ang-(1-9) lo que se asoció a mayor remodelamiento de la pared aórtica.

Previamente, hemos observado en ratas Lewis que el aumento de la presión arterial por efecto de la operación Goldblatt (GB, 2 riñones-1 pinzado, modelo experimental dependiente de AngII), se asoció a una significativa disminución de la expresión de mRNA y de la proteína ECA2 (en $-55 \%$ y $-21 \%$, respectivamente), y a menores niveles aórticos de Ang (1-9) (-25\% respecto al grupo control, 
$\mathrm{p}<0,05)$, sin diferencias en los niveles de Angs I, II y (17) ${ }^{13}$ Estos resultados sugieren que la vía paralela del SRA a través de ECA2 y Ang-(1-9) son importantes blancos para el tratamiento de la hipertensión y de sus complicaciones.

Los primeros antecedentes respecto a la participación del eje ECA2-Ang-(1-9) en el remodelamiento hipertensivo de la pared aórtica fue aportada por nuestro laboratorio. Encontramos en un modelo experimental de HTA independiente de Ang II y en ratas normotensas sham, que la inhibición de RhoA/Rho-kinasa (una vía de señalización que participa en el remodelado $\mathrm{CV}$ y renal y también en la regulación de la PA), por fasudil disminuyó la HTA, la actividad de la ECA y aumentó la actividad de ECA2 en plasma y pared aórtica. Igualmente, fasudil disminuyó los niveles plasmáticos de Ang II, aumentó los niveles de Ang-(1-9) sin modificar los niveles de Ang-(1-7), aumentó los niveles de mRNA de eNOS y disminuyó la expresión proteica de proteínas involucradas en el re- modelamiento de la pared aórtica como TGF- $\beta 1$, PAI- 1 , and MCP- 12 y también la actividad de NADPH oxidasa y producción de $\mathrm{O} 2-{ }^{28}$ Por lo tanto, el remodelamiento vascular podría ser más dependiente del eje ECA2/Ang(1-9) que de los niveles de Ang-(1-7) en ratas normotensas como hipertensas.

En conclusión, el polimorfismo de la ECA que determina mayor actividad de ECA y mayores niveles de Ang II contrarregula del eje ECA2/Ang-(1-9) lo que se asocia a mayor remodelamiento de la pared aórtica. Estas observaciones indican que nuevos e innovativos enfoques deben ser usados en el tratamiento del remodelamiento cardiovascular. En este sentido, la información disponible apunta a que el eje ECA2/Ang-(1-9) constituiría es un importante blanco para contrarrestar las acciones vasoconstrictoras y proliferativas del eje ECA/Ang II.

\section{Agradecimientos}

Este trabajo fue financiado Fondecyt 1100874

\section{Referencias:}

1. TOUYZ R. Intracellular mechanisms involved in vascular remodelling of resistance arteries in hypertension: role of angiotensin II. Exp Physiol. 2005;90: 449-455.

2. INTENGAN H, SCHIFFRIN E. Structure and Mechanica Properties of Resistance Arteries in Hypertension: Role of Adhesion Molecules and Extracellular Matrix Determinants Hypertension 2000;36:312-318.

3. MESSERLI F, WILLIAMS B, RITZ E. Essential hypertension, Lancet 2007; 370:591-603

4. TIPNIS SR, HOOPER NM, HYDE R, KARRAN E, CHRISTIE G, TURNER AJ. A human homolog of angiotensin converting enzyme. Cloning and functional expression as a captopril-insensitive cardoxypeptidase. J. Biol. Chem 2000;275: 33238-33243.

5. DONOGHUE M, HSIEH F, BARONAS E, GODBOUT $\mathrm{K}$, GOSSELIN M, STAGLIANO N, et al. A novel angiotensin-converting enzyme related carboxypeptidase (ACE2) converts angiotensin I to angiotensin 1-9. Circ. Res 2000;87: e1-e9.

6. VICKERS C, HALES P, KAUSHIK V, DICK L, GAVIN J, TANG J, et al. Hydrolisis of biological peptides by human angiotensin-converting enzyme-related carboxypeptidase (ACE2). J. Biol. Chem 2002;277: 14838-14843.

7. RICE GI, THOMAS DA, GRANT PJ, TURNER AJ, HOOPER NM. Evaluation of angiotensin-converting en- zyme (ACE), its homologue ACE2 and neprilysin in angiotensin peptide metabolism. Biochem J 2004;383:45-51.

8. SANTOS RA, CASTRO CH, GAVA E, PINHEIRO SV, ALMEIDA AP, PAULA RD, et al. Impairment of in vitro and in vivo heart function in angiotensin-(1-7) receptor MAS knockout mice. Hypertension 2006;47: 996-1002.

9. FERRARIO C. Angiotensin-converting enzyme 2 and angiotensin-(1-7): an evolving story in cardiovascular regulation. Hypertension 2006; 47: 515-521.

10. OCARANZA MP, LAVANDERO S, JALIL JE, MOYA J PINTO M, NOVOA U, et al. Angiotensin-(1-9) regulates cardiac hypertrophy in vivo and in Vitro. Journal of Hypertension 2010;28:1054-1064.

11. FLORES-MUÑOZ M, SMITH NJ, HAGGERTY C, MILLIGAN G, NICKLIN SA. Angiotensin1-9 antagonises pro-hypertrophic signaling in cardiomyocytes via the angiotensin type 2 receptor. The journal of Physiology 2011; 589.4 pp 939-951.

12. OCARANZA MP, RIVERA P, NOVOA U, PINTO M, GONZÁLEZ L, CHIONG M. RHO kinase inhibition activates the homologous angiotensin-converting enzymeangiotensin-(1-9) axis in experimental hypertension. J Hypertens. 2011;29:706-15.

13. OCARANZA M, MOYA J, PINTO M, ESCUDEROS N, VALENZUELA F, et al. Menores niveles tisulares de la enzima convertidora de angiotensina I homologa (ECA2) y angiotensina-(1-9) estan asociados a mayor remode- 
lamiento de la pared aórtica de las ratas hipertensas. Rev Chil Cardiol 2010;29:69-82.

14. OCARANZA MP, PIDDO AM, FAÚNDEZ P, LAVANDERO S, JALIL JE. Angiotensin I-converting enzyme gene polymorphism influences chronic hypertensive response in the rat Goldblatt model. J. Hypertension 2002;20:413-420.

15. GOLDBLATT H, LYNCH J, HANZAL R, SUMMERVILLE W. Studies on experimental hypertension: I. the production of persistent elevation of systolic blood pressure by means of renal ischemia. J Exp Med 1934, 59:347-379.

16. Walsh, S., Metzger, D. and Higuchi, R., ChelexR 100 as a medium for simple extraction of DNA for PCR-based typing from forensic material. Biotechniques, 1991;10:506-513.

17. RICHARDSON A, MCGOWN E, HENDERSON L, SWAN P. In vitro amino acid incorporation by the postmitochondrial supernatant from rat liver. Biochim Biophys Acta, 1971;254:468-477.

18. HILBERT P, LINDPAINTNER J, SERIKAWA T, SOUBRIER F, DUBAY C, ET AL. Chromosomal mapping of two genetic loci associated with blood-pressure regulation in hereditary hypertensive rats. Nature, 1991;353:521-529

19. OCARANZA MP, GODOY I, JALIL JE, VARAS M, COLLANTES P, PINTO M, et al. Enalalpril attenuates downregulation of angiotensin-converrung enzyme 2 in the late phase of ventricular dysfunction in myocardial infracted rat. Hypertension 2006, 48:572-578.

20. OLIVERI C, OCARANZA MP, CAMPOS X, LAVANDERO S, JALIL JE. Angiotensin I-converting enzyme modulates neutral endopeptidase activity in the rat. Hypertension 2001; 38: 650-654.

21. JOHNSON G, AHN K. Development of an internally quenched fluorescent substrate selective for endothelinconverting enzyme-1. Anal. Biochem 2000; 286: 112-118.

22. LAWRENCE AC, EVIN G, KLADIS A, CAMPBELL DJ. An alternative strategy for the radioimmunoassay of angiotensin peptides using amino-terminal-directed antisera: measurement of eight angiotensin peptides in human plasma. J Hypertens 1990; 8: 715-724.

23. IGASE M, STRAWN WB, GALLAGHER PE, GEARY RL, FERRARIO CM. Angiotensin II AT1 receptors regulate ACE2 and angiotensin-(1-7) expression in the aorta of spontaneously hypertensive rats. Am J Physiol Heart Circ Physiol 2005; 289:H1013-H1019.

24. RIVERA P, OCARANZA MP, LAVANDERO S, JALIL JE. Rho Kinase Activation and Gene Expression Related to Vascular Remodeling in Normotensive Rats With High Angiotensin I Converting Enzyme Levels. Hypertension 2007; 50:792-798.

25. BRADFORD M. A rapid and sensitive method for the cuantitation of microgram cuantities of protein utilizing the principle of protein-dye binding. Anal. Biochem 1976;
$72: 248-254$

26. SALVADÓ M, MARTÍNEZ S, ÁLVARO T, BARBERA

M, RISA R, et al. Técnicas de desenmascaramiento antigénico en inmunohistoquímica. Rev Esp Patol 2001; 34:255260.

27. LIU J, YANG F, YANG XP, JANKOWSKI M, PAGANO PJ. NAD(P)H Oxidase Mediates Angiotensin II-Induced Vascular Macrophage Infiltration and Medial Hypertrophy. Arterioscler Thromb Vasc Biol 2003; 23:776-782.

28. JALIL JE, PÉREZ A, OCARANZA MP, BARGETTO J, GALAZ A, LAVANDERO S. Increased aortic NADPH oxidase activity in rats with genetically high angiotensinconverting enzyme levels. Hypertension 2005; 46:13621367.

29 IWAI M, HORIUCHI M. Devil and angel in the renin-angiotensin system: ACE-angiotensin II-AT1 receptor axis vs ACE2-angiotensin-(1-7)-Mas receptor axis. Hypertension Research 2009; 32:533-536.

30. LACHURIÉ M, AZIZI M, GUYENE T, ALHENC-GELAS F, MÉNARD J. Angiotensin-converting enzyme gene polymorphism has no influence on the circulating reninangiotensin-aldosterone system or blood pressure in normotensive subjects. Circulation 1995;91:2933-2342.

31. BERGE K, BERG K. No effect of insertion/deletion polymorphism at the ACE locus on normal blood pressure level or variability. Clin Genet 1994; 45:169-174.

32. O'DONNELL C, LINDPAINTNER K, LARSON M, RAO $\mathrm{V}$, ORDOVAS J, et al. Evidence for association and genetic linkage of the angiotensin-converting enzyme locus with hypertension and blood pressure in men but not women in the Framingham Heart Study. Circulation 1998; 97:1766 -1772 .

33. CHALLAH M, VILLARD E, PHILIPPE M, RIBADEAUDUMAS A, GIRAUDEAU B, JANIAK P, et al. Angiotensin I-converting enzyme genotype influences arterial response to injury in normotensive rats. Arterioscler Thromb Vasc Biol 1998, 182:235-243.

34. PARKER SB, WADE SS, PREWITT RL. Pressure Mediates Angiotensin II-Induced Arterial Hypertrophy and PDGF-A. Hypertension 1998, 32:452-458.

35. RUPÉREZ M, LORENZO O, BLANCO-COLIO LM, ESTEBAN V, EGIDO J, RUIZ-ORTEGA M. Connective tissue growth factor is a mediator of angiotensin II-induced fibrosis. Circulation 2003; 108, 1499-1505.

36. ZHAO Q, ISHIBASHI M, HIASA K, TAN C, TAKESHITA A, et al. Essential role of vascular endothelial growth factor in angiotensin II-induced vascular inflammation and remodeling. Hypertension 2004; 44:264-270.

37. TOUYZ RM, BERRY C. Recent advances in angiotensin II signaling. Braz J Med Biol Res 2002; 35:1001-1015.

38. DZAU V, BERNSTEIN K, CELERMAJER D, COHEN J, 
DAHLOF B, DEANFIELD J, et al. Pathophysiologic and therapeutic importance of tissue ACE: a consensus report. Cardiovasc Drugs Ther 2002;16:149-160.

39. SILVA D, VIANNA H, CORTES S, CAMPAGNOLESANTOS M, SANTOS R, LEMOS VS. Evidence for a new angiotensin-(1-7) receptor subtype in the aorta of Sprague-Dawley rats. Peptides 2007;28:702-707.

40. WEISS D, BERNSTEIN K, FUCHS S, ADAMS J, SYNETOS A, TAYLOR W. Vascular wall ACE is not required for atherogenesis in ApoE-/- mice. Atherosclerosis 2010; 209:352-358.

41. LEE R, BADER M, ALENINA N, SANTOS R, GAO Y, LU C. Mas receptors in modulating relaxation induced by perivascular adipose tissue. Life Sci 2011; 89:467-472

42. LEE R, LU C, SU L, GAO Y. Endothelium-dependent relaxation factor released by perivascular adipose tissue. $\mathrm{J}$ Hypertens 2009; 27:782-790.

43. SAMPAIO W, HENRIQUE DE CASTRO C, SANTOS R, SCHIFFRIN E, TOUYZ R. Angiotensin-(1-7) counterregulates angiotensin II signaling in human endothelial cells. Hypertension 2007; 50:1093-1098.

44. SAMPAIO W, SOUZA DOS SANTOS R, FARIA-SILVA
R, DA MATA MACHADO L, SCHIFFRIN E, TOUYZ R. Angiotensin-(1-7) through receptor Mas mediates endothelial nitric oxide synthase activation via Akt-dependent pathways. Hypertension 2007; 49:185-192.

45. RENTZSCH B, TODIRAS M, ILIESCU R, POPOVA E, CAMPOS L, OLIVEIRA M, et al. Transgenic angiotensin-converting enzyme 2 overexpression in vessels of SHRSP rats reduces blood pressure and improves endothelial function. Hypertension 2008; 52:967-973.

46. RABELO L, ALENINA N, BADER M. ACE2-angiotensin-(1-7)-Mas axis and oxidative stress in cardiovascular disease. Hypertens Res 2011; 34:154-160

47. ESTEBAN V, HERINGER-WALTHER S, STERNERKOCK A, DE BRUIN R, VAN DEN ENGEL S, WANG $\mathrm{Y}$, et al. Angiotensin-(1-7) and the g protein-coupled receptor MAS are key players in renal inflammation. PLoS One 2009; 4:e5406.

48. VELKOSKA E, DEAN R, GRIGGS K, BURCHILL L, BURRELL LM. Angiotensin-(1-7) infusion is associated with increased blood pressure and adverse cardiac remodelling in rats with subtotal nephrectomy. Clin Sci 2011; 120:335-345. 